\title{
BLOCKCHAIN: SOCIAL INNOVATION IN FINANCE \& ACCOUNTING
}

\author{
Reepu \\ Assistant Professor, University School of Business \\ Chandigarh University, Gharuan, India
}

\begin{abstract}
At present times, there has been a surge in cryptopians community. Since wealth maximization is the aim of many, the virtual currency has become one of the important investment avenues. Virtual currency like bitcoins have become grill for the gossip mill. Consumer enjoys sovereignty. The present paper provides insight of the behind the scene technology in order to attain better understanding of the securitization prospects pertaining to the same. Mining allures cryptopians to solve proof of work because then only an increment in rewarded in blockchain. Without a doubt as per statistics, the currency has seen an upsurge but vital determinants which may pose a threat needs to be taken care of
\end{abstract}

Keywords: Bitcoin, Blockchain, Cryptopians etc.

Cite this Article: Reepu, Blockchain: Social Innovation in Finance \& Accounting, International Journal of Management, 10 (1), 2019, pp. 14-18.

http://iaeme.com/Home/issue/IJM?Volume=10\&Issue=1

\section{INTRODUCTION}

Blockchain, a cornerstone in financial market, assures investment in bitcoins, Ethereum, bitcoin cash and stellar lumens through the usage of most secured platform bitcoin wallet. Claimant says that there are over 32 million wallets and onto which $\$ 200 \mathrm{~B}$ have been invested. There is no middle man in bitcoin transaction, hence blockchain allows peer to peer bitcoins transactions. Bitcoin is divisible and portable. It minimizes cost and power lies in the hand of consumer. Consumer may prefer bitcoin transactions as:

- It outperformed over the last five years.

- Cross border transactions are permissible.

- Safekeeping in non-custodial wallet.

- Entire globe is a market.

Ether another intelligent digital avenue, empowers network by paying transactional fees. This fee is paid to miners. Ether has more potential applications in medicines, global supply chains etc. Therefore, ether serves futurity. 


\section{REVIEW OF LITERATURE}

Eyal I and Sirer E.G. (2013) found that bitcoin protocol is not at all incentive compatible. They presented in their research that miners collect majority revenue share than designated to them. Joancomarti J.H. (2014) believed that anonymity and decentralization are the stepping stone of bitcoin's success. The paper results into research challenges on cryptocurrency. Dyhrberg A.H (2015) researched about bitcoin's hedging capabilities through the application of asymmetric GARCH methodology. Research concluded that bitcoin can be used to hedge against American Dollar for shorter time period as well as FTSE Index. Holub M. and Johnson J. (2018) have mapped bitcoin across six discipline viz. technical fields, economics, law, public policy, finance and accounting after a review of 1,206 papers. Nakamoto S. (2018) believed that bitcoins enable sending money peer to peer through network timestamps but with the recording of proof-ofwork. The dialogues across the network are secured through mining.

\section{BLOCKCHAIN}

Blockchain, the buzzword behind bitcoin, is a public ledger that is distributed over different nodes. Each block pertains specific information stamp and all of them combined together forms a chain. Blocks therefore comprises:

1. Date, Time and Amount specific information.

2. Transaction participants

3. A unique hash code that distinguishes one block from other.

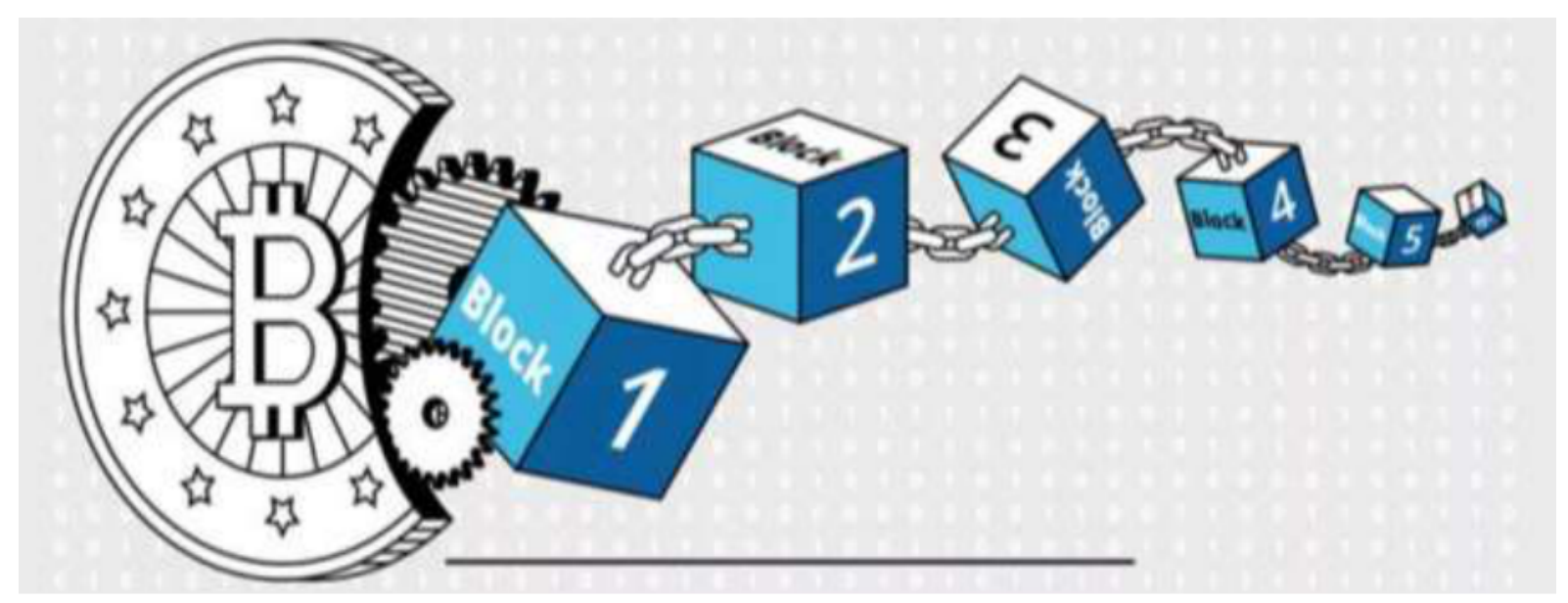

Figure 1 Source: Investopedia

\subsection{Working:}

In order to frame information hawser a transaction must transpire. The authentication of transaction details in this case, is directed by computer network who verify the purchase details so that the confirmation can be laid. It comes in green area as soon as it gets approval and with the consequence of that the corresponding digital signatures are stowed in a specific block and then the chain will be formulated by combining the different blocks. Finally, the block is designated with a hash. 


\subsection{Technique behind blockchain}

Consider any website for instance, XYZ. Both XYZ and blockchain glide on distributed platform but XYZ utilizes traditional Client-Server methodology. Any client therefore with due authorization, whenever introduce changes they are hoarded on centralised server only. Hence any other user who know accesses that website will see the updated page from the master copy only.

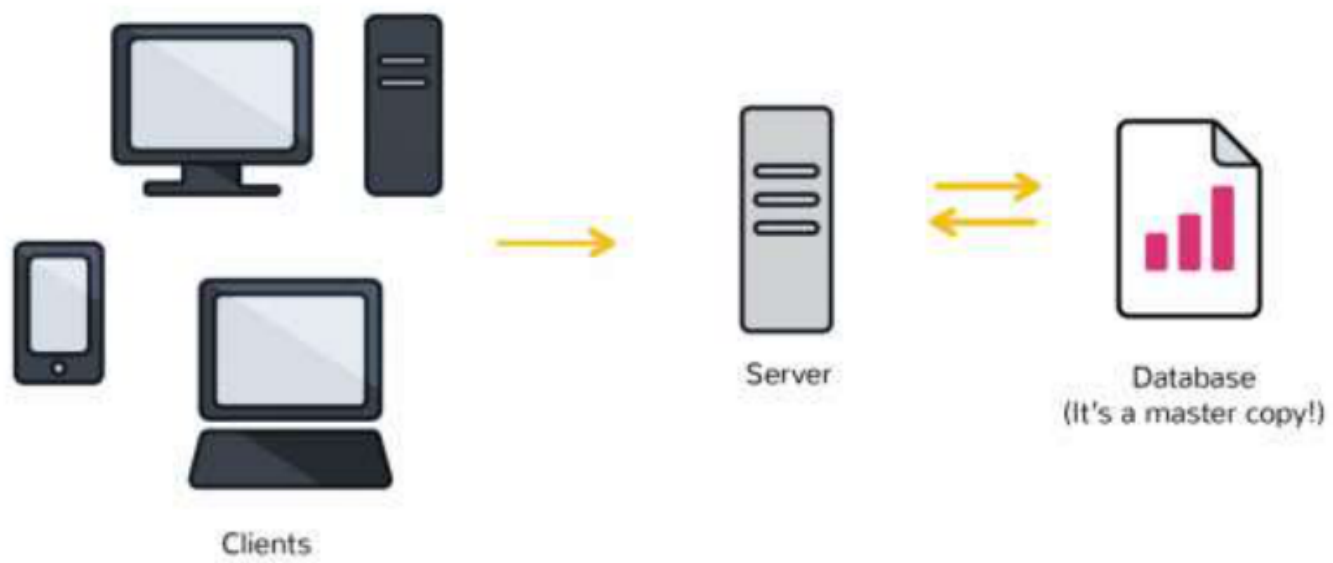

Figure 2 Source: Coindesk

From the above instance it can be inferred that this traditional Client Server Model is the dome of application in banks, insurance companies etc. Here the data manipulation lies with few personnel only.

Blockchain on the other hand, are diametrically different than Client-Server model. There XYZs updated master copy was visible to the users who wish to access it. But in case of blockchain every node is clinching towards the same end, thereby making upgradations then and there.

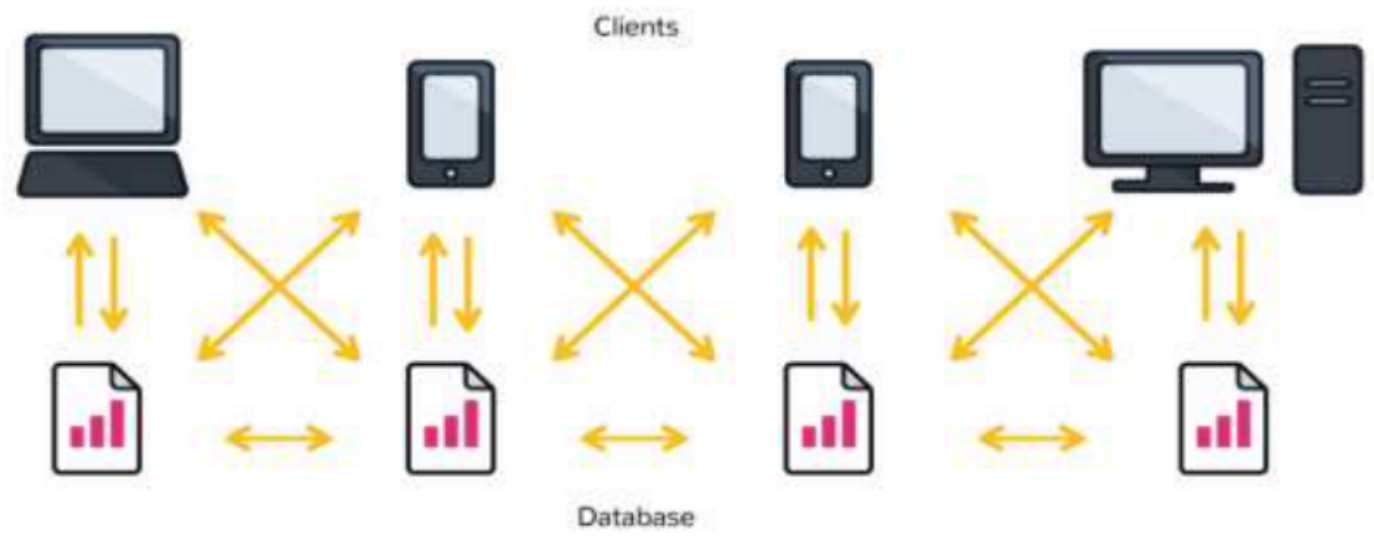

Figure 3 Source: Coindesk 
Hence in Blockchain, each existing node in chains updates the relevant information. It deploys technologies viz. Internet, Cryptography and protocol emphasizing incentivization.

\subsection{Important points:}

Blockchain

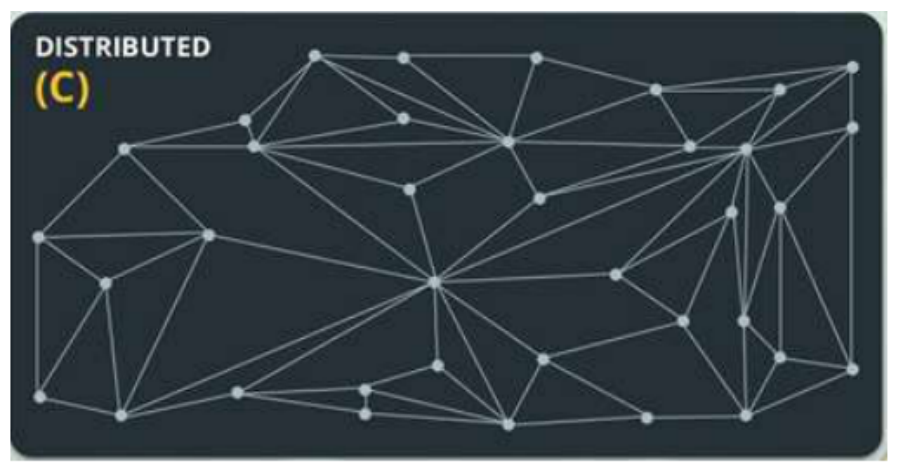

Figure 4. (a) Distributes

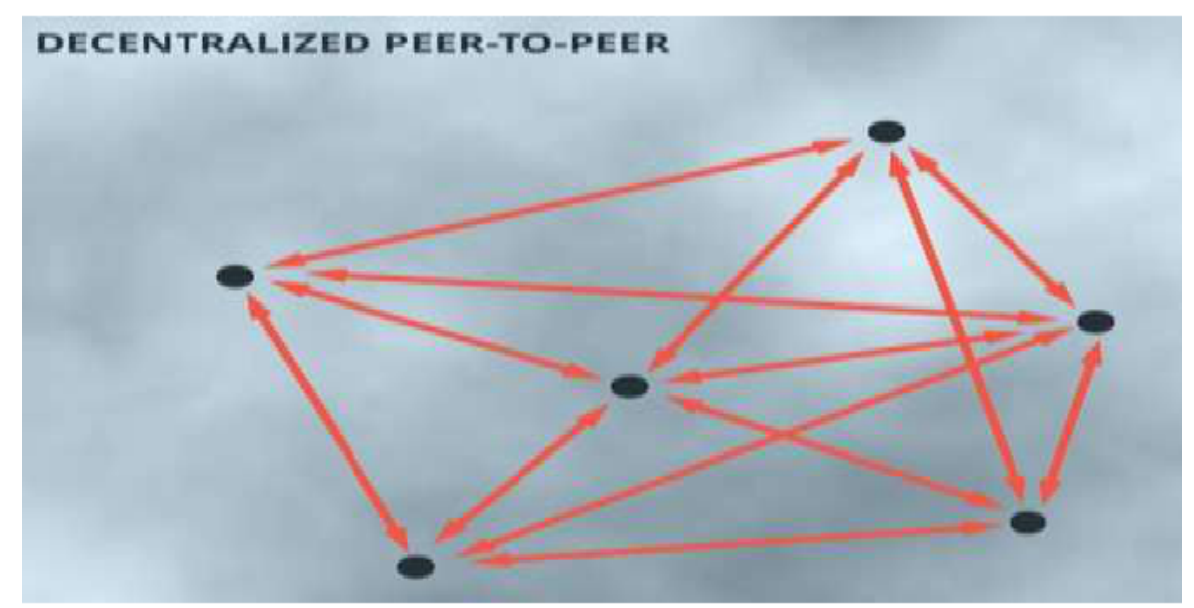

Figure 5. (b) Decentralises

(c) Transparent but maintains anonymity- data is accessible with hash value only

\subsection{Bitcoin}

Bitcoins pertains bitcoin wallet which has bitcoin address. Anyone who wishes to transact would make use of that bitcoin address. Bitcoin utilizes blockchain technology to effectuate transfers. Bitcoin wallets keep some private key which is used to authenticate transactions. Nobody can manipulate signed transaction. Transactions after signatures, are broadcasted after 10-20 minutes, with the help of mining.

Mining: Any pending transaction is rolled in block chain with the help of mining. Networks are neutralized. Transactions are jampacked with cryptographic rules hence manipulation becomes tedious. Moreover, it's just mining because of which new bitcoins are issued.

Once the transaction is hashed, then in order to attach a new block to the chain there is requisition of proof of work to be attached i.e. actually assuring that new block is tedious to add. Mining is rewarded as shown below: 


\section{Reepu}

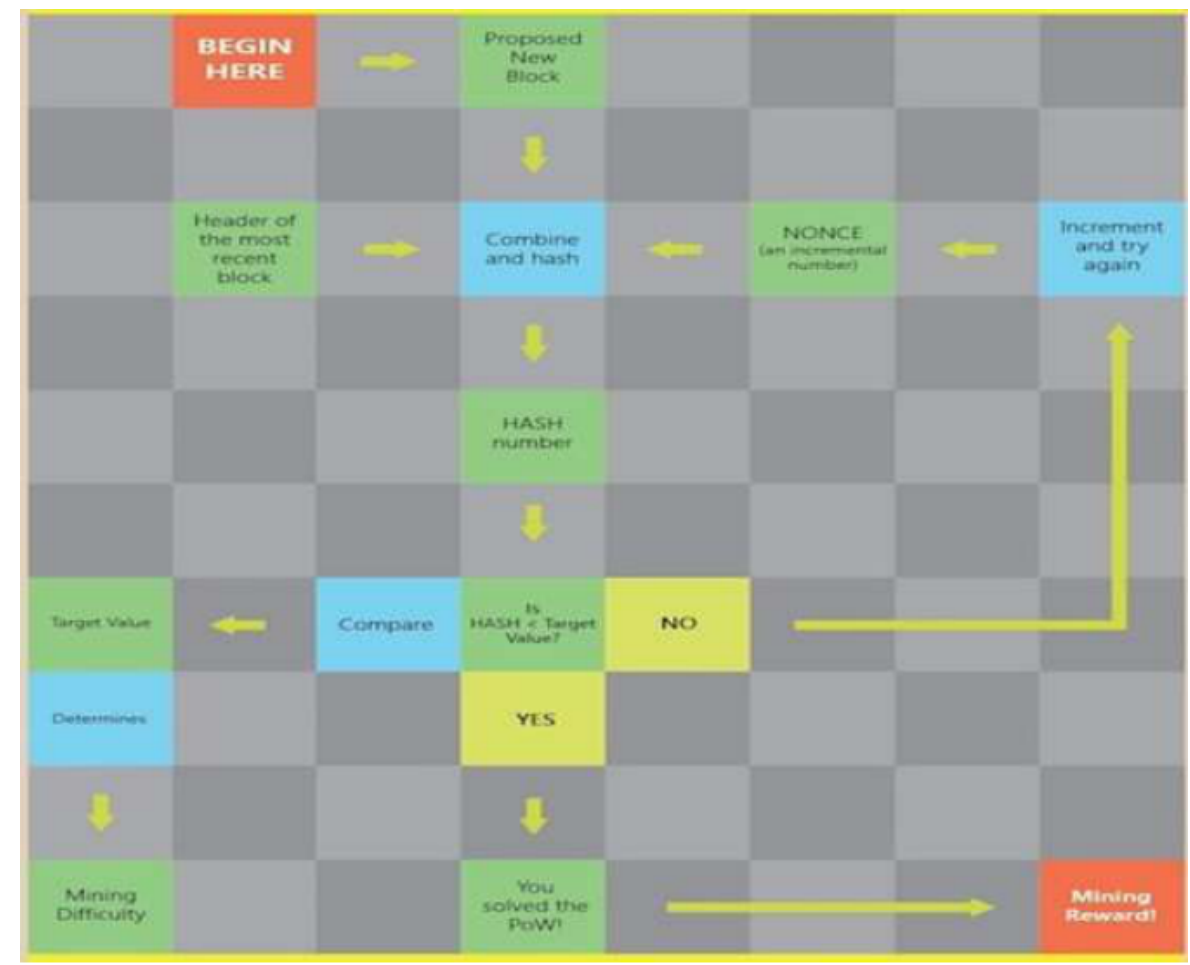

Figure 6 Source: Bitcoin Mining

As shown in the diagram, as soon as new block is proposed, it is deployed with a hash. Now the cryptopians need to work on proof on work. If the problem is solved viz. the hash value is less than the target value, mining is rewarded and finally block is rewarded. Otherwise the value is not incremented, and reworking is desired.

\section{CONCLUSION}

Transacting virtual currency that too peer to peer is an asset for an investor. Such cryptocurrency transactions ensure delivery through a secured cryptographic platform. Now-adays bitcoins have been in limelight since they have outperformed over the last few years. Numerous others like Ethereum, stellar lumens etc are also transacted. The present paper discusses the beyond technology in order to understand the security prospects of the same as few predicts that 2019 can be the year of attack.

\section{REFERENCES}

[1] Eyal, Ittay \& Sirer, Emin, Majority Is Not Enough: Bitcoin Mining Is Vulnerable. 8437, 2013.

[2] Joancomarti J.H. Research and Challenges on Bitcoin Anonymity, 9th International Workshop on Data Privacy Management. Springer LNCS 8872,2014, pp.1-14

[3] Manisha Valera, Parth Patel and Shruti Chettiar, an Avant-Garde Approach of Block chain in Big Data Analytics, International Journal of Computer Engineering and Technology, 9(6), 2018, pp. (115)-(120).

[4] Dyhrberg A.H, Hedging Capabilities of Bitcoin. Is it the Virtual gold? UCD School of Economics, University College Dublin, Belfield Dublin 4, 2015

[5] Holub M. and Johnson J. Bitcoin Research across disciplines, The Information Society, Vol.34, No.2, 2018, pp 114-126.

[6] Dr. Sanjeev Kumar, Sanchita Raghav, Apoorva Raj, Ankit Tiwari and Mohan Gautam, Social Innovation and Social Entrepreneurship - An Elucidation for the Problems of Modern Society. International Journal of Management, 8(1), 2017, pp. 212-217.

[7] Nakamoto S, Bitcoin: A peer-to-peer Electronic Cash System, www.bitcoin.org, 2018 